\section{Pénfigo vulgar tipo cutáneo. Caso clínico}

\author{
CRISTINA BELLO ${ }^{1}$, LUIS MONDACA-CORNEJO ${ }^{1}$, \\ CRISTIÁN NAVARRETE-DECHENT ${ }^{1}$, SERGIO GONZÁLEZ ${ }^{2}$
}

\section{Cutaneous pemphigus vulgaris. A case report}

Pemphigus is an autoimmune bullous disease that involves skin and mucous membranes caused by autoantibodies against antigens on the surface of keratinocytes. We report a 30-year-old male presenting with a five months history of pruriginous alopecic and crusted lesions in the scalp, that extended posteriorly to the trunk and limbs. Mucous membranes were not involved. A skin biopsy was performed, showing extensive loss of epidermis and acantholysis. Immunofluorescence was positive for C3 and intercellular and epidermic IgG. With the presumptive diagnosis of pemphigus vulgaris $(P V)$ without mucous involvement, the patient was treated with prednisone, observing an excellent clinical response. There are only few cases published in the literature of $P V$ without mucous involvement. Some authors refer to this subtype of $P V$ as "Cutaneous pemphigus vulgaris".

(Rev Med Chile 2013; 141: 525-530).

Key words: Acantholysis; Desmogleins; Pemphigus.

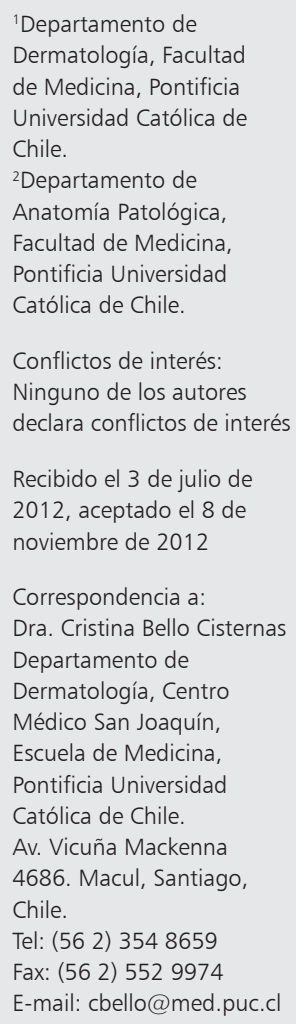

$\mathrm{E}$ 1 pénfigo es una enfermedad ampollar autoinmune que compromete piel y mucosas ${ }^{1}$. Tiene una incidencia anual variable de país a país de aproximadamente $0,75-5$ casos por millón de habitantes ${ }^{1}$. La enfermedad es causada por autoanticuerpos contra antígenos de la superficie de los queratinocitos causando acantólisis²

Existen dos formas clásicas de pénfigo, dependiendo el nivel de compromiso epidérmico: el pénfigo vulgar (PV) y el pénfigo foliáceo (PF). Se afectan diferentes capas epidérmicas, causan síntomas diferentes y son producidos por autoanticuerpos contra antígenos distintos ${ }^{1}$. Otras variantes del pénfigo incluyen el pénfigo vegetante, el pénfigo eritematoso, el pénfigo foliáceo endémico (fogo salvagem $)^{3}$, el pénfigo paraneoplásico, el pénfigo inducido por drogas ${ }^{1} \mathrm{y}$ el pénfigo por IgA.

El PV se puede desarrollar a cualquier edad pero es más frecuente entra la cuarta y sexta década de vida. No tiene diferencias por $\operatorname{sexo}^{4}$ y se encontraría con mayor prevalencia en las personas con ascendencia mediterránea ${ }^{1}$ o judía ${ }^{5}$. La enfermedad se inicia habitualmente con úlceras orales dolorosas que no curan ${ }^{6}$, a diferencia de las virales o de la estomatitis aftosa, que curan en días o semanas ${ }^{1}$. Rara vez se encuentran ampollas en las mucosas debido a que se rompen precozmente. Las úlceras son múltiples, superficiales e irregulares y se originan en mucosas sanas. Las mucosas más comúnmente comprometidas son la labial, palatina y de la lengua. Por esta razón, el diagnóstico de pénfigo debe considerarse en toda úlcera que no cure en un período mayor a un mes ${ }^{1}$. Luego de semanas a meses, comienza el compromiso cutáneo, inicialmente en cuero cabelludo, cara y tórax superior pudiendo acompañarse de síntomas de compromiso nasal y esofágico. La enfermedad puede también comprometer la región periungueal ${ }^{1}$.

Las lesiones cutáneas se originan en piel sana, inicialmente como ampollas flácidas, que se rompen durante los primeros días transformándose en erosiones superficiales con un collarete de epidermis laxa alrededor ${ }^{1}$. El PV activo -y en sus formas más severas-, presenta signo de Nikolsky positivo en piel sana y perilesional. 
El compromiso cutáneo sin compromiso mucoso en el caso de un PV es bastante infrecuente ${ }^{1}$. Presentamos el caso de un paciente con PV con compromiso cutáneo en ausencia de compromiso mucoso y una revisión de la literatura.

\section{Caso clínico}

Paciente de 30 años de sexo masculino sin antecedentes mórbidos ni historia de consumo de fármacos. Consultó por un cuadro de 5 meses de evolución, de placas alopécicas pruriginosas y costrosas en cuero cabelludo, con extensión posterior de erosiones costrosas a tronco y extremidades (Figura 1 y 2). Se realizó cultivo de hongos de lesiones que resultó positivo para T. rubrum.

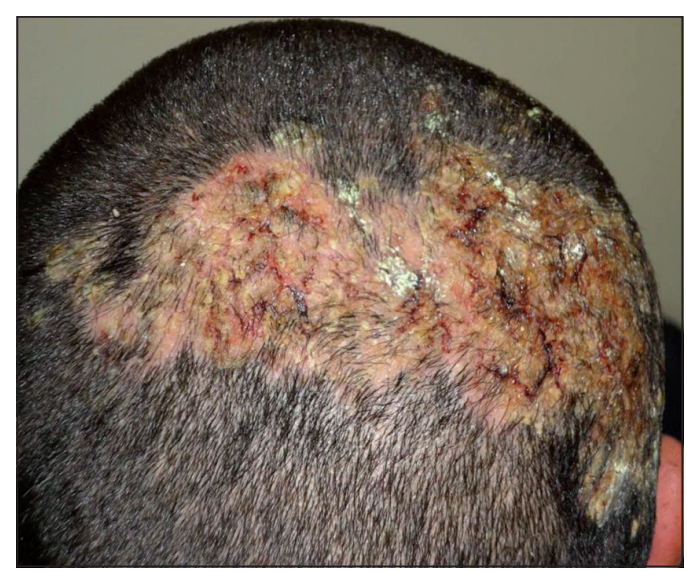

Figura 1. Extensa placa alopécica erosiva en cuero cabelludo con costras hemáticas.

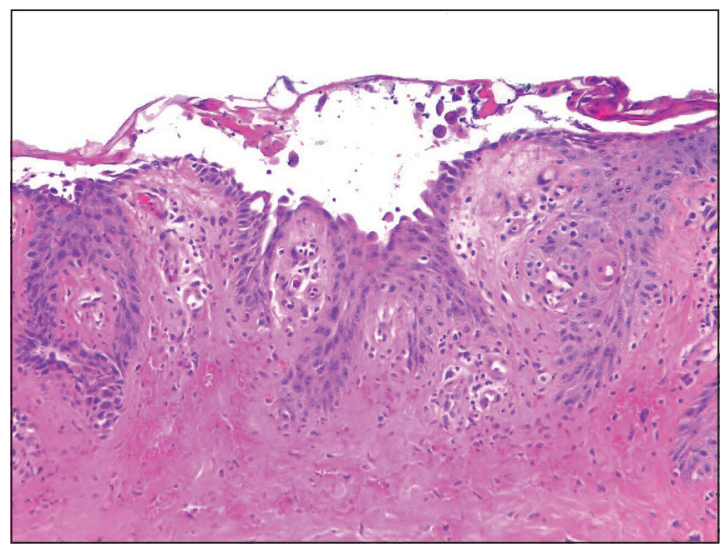

Figura 3. Extensa pérdida de epidermis por acantólisis de ampolla intraepidérmica, suprabasal (H\&E 20X).
Recibió inicialmente ciprofloxacino, mupirocina y terbinafina tópica con mala respuesta. Se inició luego terbinafina oral y prednisona por diagnóstico presuntivo de tiña capitis inflamatoria.

Se controló a las 2 semanas destacando en pierna y dorso de pie nueva erosión con superficie destechada sin presentar compromiso de mucosas. Al laboratorio destacaba: leucocitosis de 12.500 células $/ \mathrm{mm}^{3} \sin$ desviación a izquierda y VHS 14 mmHr.

Por sospecha de enfermedad ampollar se solicitó biopsia de piel con inmunofluorescencia directa (IFD) la cual mostró extensa pérdida de epidermis por acantólisis de ampolla intraepidérmica, suprabasal (Figura 3). La IFD resultó positiva para C3 e IgG intercelular y epidérmico, y negativa para IgA, IgM y fibrina (Figura 4).

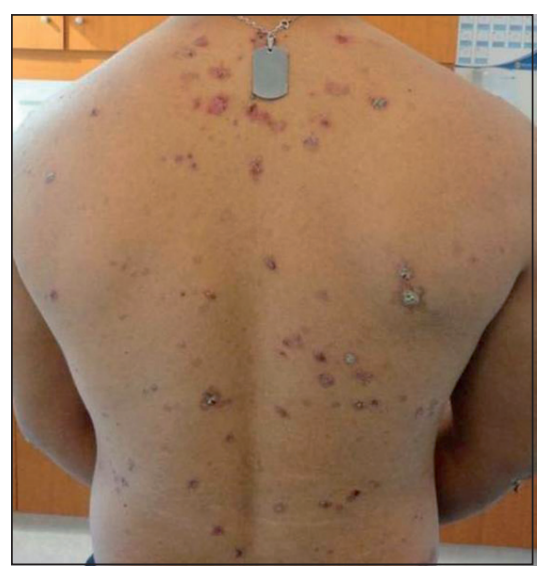

Figura 2. Múltiples placas y pápulas erosivas con costras hemáticas en dorso.

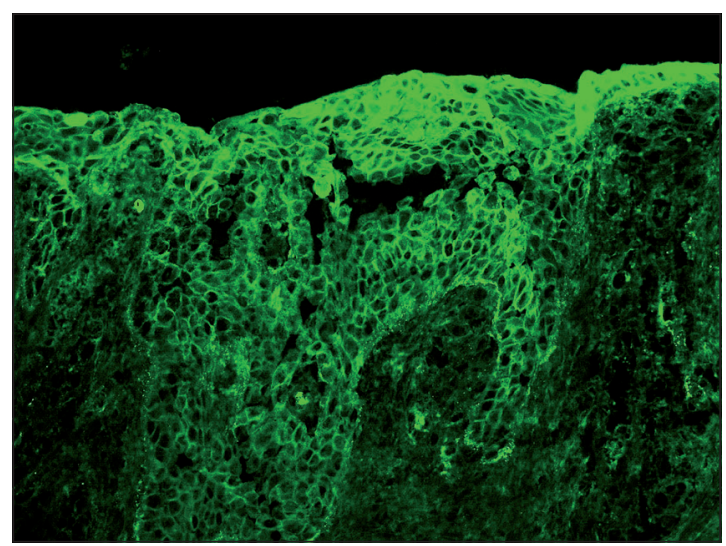

Figura 4. Inmunofluorescencia directa positiva contra IgG en patrón intercelular. 
Los hallazgos clínicos y de laboratorio fueron compatibles con pénfigo vulgar sin compromiso de mucosas. Se inició tratamiento con prednisona $1 \mathrm{mg} / \mathrm{kg} /$ día por 30 días con excelente respuesta y repoblación capilar en cuero cabelludo. Se realizó disminución gradual de prednisona, entrando en fase de mantención sin reaparición de lesiones.

\section{Discusión}

El PV es la variante más frecuente de pénfigo $(70-90 \%)^{4,7}$ y se considera una variante de compromiso epidérmico profundo ${ }^{4}$. El compromiso cutáneo sin compromiso mucoso, como en nuestro caso, ha sido reportado excepcionalmente, existiendo sólo 8 casos publicados en la literatura lo cual pudo contribuir a la demora en el diagnóstico definitivo ${ }^{8,9}$.

Este subtipo de PV también se ha llamado "Pénfigo vulgar tipo cutáneo" sin quedar completamente aclarado si este tipo de PV pudiese ser un estado transitorio antes de desarrollar compromiso mucoso o se tratará de un subgrupo aparte. Por otro lado, el compromiso de cuero cabelludo y cara como lesión inicial también es infrecuente en los PV mucocutáneos y ocurriendo en 15\% de los pacientes ${ }^{10}$, lo que pudo haber sido otro factor que bajó nuestra sospecha diagnóstica.

Es característica de todos los pénfigos la separación de los queratinocitos o acantólisis ${ }^{1}$. El $80 \%$ de los pénfigos cursan con anticuerpos ( $\mathrm{IgG}$ ) circulantes en las etapas activas de la enfermedad, cuyos títulos se correlacionan con la actividad de la misma. Los anticuerpos son patogénicos y tienen la capacidad de inducir acantolisis in vitro en cultivos de piel humana ${ }^{11}$. Las moléculas de adhesión de los queratinocitos llamadas desmogleínas (Dsg) son el blanco de los autoanticuerpos, siendo las más caracterizadas las Dsgs1 y 3. Ambas Dsgs son proteínas de transmembrana del desmosoma y pertenecen al grupo de las caderinas ${ }^{12}$.

El PV sin compromiso mucoso se podría explicar por la teoría de compensación de las Dsgs (Figura 5). En el PV se producen mayoritariamente autoanticuerpos contra la Dsg 3, expresada en la superficie de los queratinocitos y en mucosas, con un mayor porcentaje de expresión hacia la región basal (suprabasal) que hacia la región superficial (subcórnea) de la epidermis ${ }^{1}$. Las mucosas expresan Dsg3 en abundante cantidad ${ }^{1}$. En el PF hay anticuerpos contra Dsg1, causando lesiones sólo en la epidermis superficial, donde la Dsg1 se expresa mayoritariamente, sin co-expresión de Dsg3. En la epidermis profunda la presencia de Dsg3 compensa la pérdida de función de Dsg1. Aunque se liguen anticuerpos a Dsg1 en las mucosas, no se forman ampollas debido a la fuerte co-expresión de Dsg3 en todo el espesor epidémico ${ }^{8}$. Esto explicaría las ampollas superficiales del PF.

Debido a que la expresión de Dsg3 es mucho menor en la piel que en la mucosa oral, el PV limitado a la piel, podría deberse a expresión de anticuerpos anti Dsg3 débiles, que pudiesen ser lo suficientemente fuertes para bloquear la Dsg3 de la piel, pero no lo suficientemente potentes para bloquear la función de la Dsg3 en las mucosas,

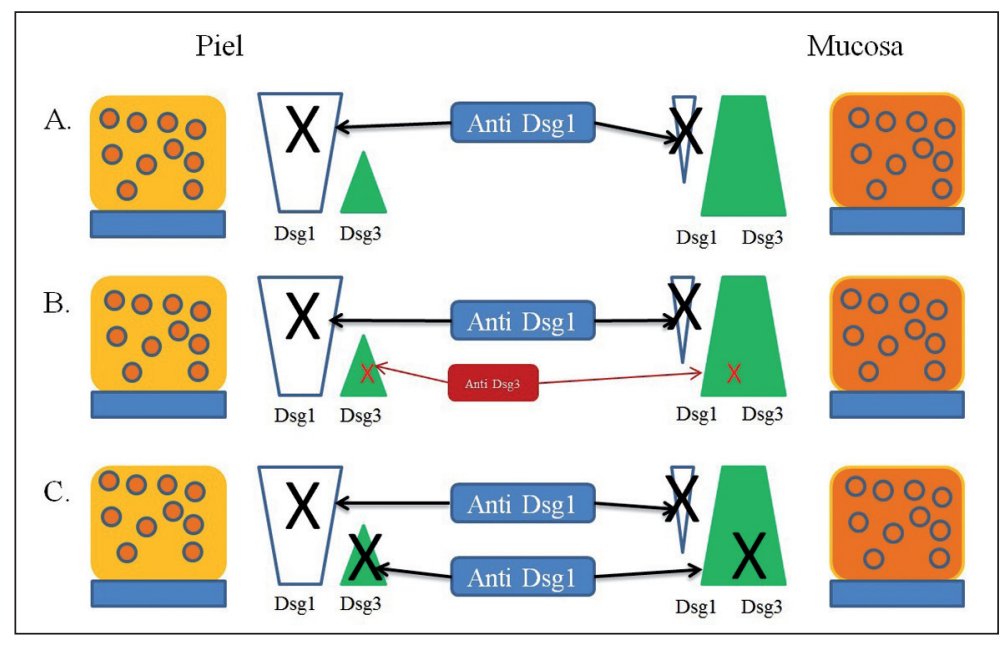

Figura 5. Teoría de la expresión de desmogleínas (Dsg). Los triángulos y trapezoides expresan el nivel de expresión de Dsg1 (blanco) y Dsg3 (verde) en la piel (columna izquierda) y en las mucosas (columna derecha). El tamaño de los rectángulos de los anticuerpos indica la potencia patogénica para formar ampollas. A: Pénfigo foliáceo. B: Pénfigo vulgar limitado a la piel. C: Pénfigo vulgar mucocutáneo. En "B" hay anticuerpos patogénicos débiles contra Dsg3 y potentes anti-Dsg1. 
como ocurre en el PV clásico ${ }^{8,9}$. Estos anticuerpos "débiles", logran generar ampollas suprabasales en presencia de anticuerpos anti Dsg1, debido a que las uniones desmosómicas basales se consideran físicamente más débiles que las uniones de las capas superiores. Por lo tanto, la combinación de anticuerpos débiles anti Dsg3 y potentes anti Dsg1 podría explicar el PV limitado a la piel ${ }^{8}$.

Últimamente, se ha visto que los pacientes con pénfigo desarrollan autoanticuerpos contra otras proteínas; $85 \%$ de los pacientes con pénfigo desarrollan anticuerpos contra la porción $9 \alpha$ del receptor nicotínico de acetilcolina, los cuales también podrían producir acantolisis. Esta unión bloquearía el ingreso de calcio necesario para el ensamblaje del desmosoma, lo cual debilitaría las uniones entre queratinocitos, causando acantólisis $^{13}$.

El diagnóstico de PV se realiza con la clínica, la histología e inmunohistoquímica. En los últimos años se ha agregado un tercer pilar de diagnóstico que consiste en los test serológicos.

El estudio histopatológico muestra una ampolla intraepidérmica con acantólisis; en el caso del PV la ampolla está justo por encima de las células basales. El examen más importante y considerado gold standard para el diagnóstico de pénfigo es la IFD, la cual mostrará depósitos de inmunoglobulinas intercelulares o "en encaje" en la epidermis ${ }^{1}$.

Se ha intentado realizar IFD en test de Tzanck. Algunos estudios han mostrado que sería igualmente sensible a realizar IFD en biopsia convencional en los primeros 3 meses desde el inicio de la enfermedad; luego de este período la sensibilidad caería en forma considerable (20\% versus $100 \%)^{14}$.

La detección de anticuerpos circulantes puede hacerse mediante inmunofluorescencia indirecta o mediante ELISA contra Dsgs1 y 3. La detección de Dsg3 mediante ELISA en PV tiene una sensibilidad de $90,8 \%$ y una especificidad de $98,7 \%{ }^{15}$. La detección de autoanticuerpos por ELISA tendría algunas ventajas como requerir menos tiempo y ser más rápido que el diagnóstico por $\operatorname{IFD}^{16}$. Sin embargo, debido a la gravedad de la enfermedad, y a que se requerirá el uso de tratamientos prolongados y no exentos de efectos adversos, el diagnóstico debe aún ser confirmado mediante histología con IFD ${ }^{1}$.

Antes del advenimiento de los corticoides, el pénfigo era una enfermedad casi invariablemente fatal ${ }^{1}$. En un estudio retrospectivo de causa de mortalidad en pacientes hospitalizados por enfermedades dermatológicas entre 1995-2001; el pénfigo fue la causa más frecuente, explicando $35 \%$ de todas las causas (tasa de mortalidad global $3,58 \%)$. Todos estos pacientes tenían más de 70\% de compromiso cutáneo y las causas de muerte fueron compromiso cutáneo extenso, sepsis, neumonía y alteraciones hidroelectrolíticas ${ }^{17}$.

El diagnóstico diferencial del PV debe considerar el penfigoide buloso, la necrolísis epidérmica tóxica, el Síndrome de Steven-Johnson, otras erupciones por fármacos, quemaduras, otras variantes de pénfigo como el pénfigo por IgA y las estomatitis por quimioterapia, entre otros.

La piedra angular del tratamiento del PV son los corticoides por vía oral o intravenosa en pulsos, usualmente con combinación con algún ahorrador de corticoides. Se recomienda iniciar el tratamiento con prednisona $1-2 \mathrm{mg} / \mathrm{kg} / \mathrm{día}$, o dosis equivalente, para luego ir reduciendo gradualmente la dosis.

El tratamiento tiene tres etapas: fase de control, fase consolidación y fase de mantención. La primera etapa tiene por finalidad controlar rápidamente la enfermedad, evitando la aparición de nuevas lesiones; la segunda etapa tiene por finalidad mantener el tratamiento hasta que hayan curado $80 \%$ de las lesiones. La tercera etapa consiste en disminuir la dosis de los tratamientos -al mínimo necesario-, para prevenir la aparición de nuevas lesiones ${ }^{1}$. El objetivo de esta tercera etapa sería discontinuar definitivamente el tratamiento.

Una revisión sistemática reciente no logró demostrar la superioridad de algún tratamiento sobre otro ${ }^{18}$. Sin embargo, otra revisión sistemática mostró que el mejor ahorrador de corticoides pareciera ser la azatioprina en dosis de $2-3 \mathrm{mg} / \mathrm{kg} / \mathrm{d}$, aunque el mofetil micofenolato podría ser similar o levemente inferior como agente ahorrador de corticoides que la azatioprina. Este estudio mostró que no tendría beneficios adicionales agregar pulsos de dexametasona al tratamiento con prednisona y azatioprina ${ }^{19}$.

Otros tratamientos que han mostrado efectividad son el metrotrexato, la ciclofosfamida y la plasmaféresis.

La inmunoglobulina intravenosa en dosis de $2 \mathrm{gr} / \mathrm{kg} /$ día por 3 a 5 días cada 4 sem sería útil en casos resistentes a tratamiento habitual, debido a que disminuye selectiva y rápidamente los niveles circulantes de autoanticuerpos. 
El uso de rituximab, un anticuerpo anti CD20 que causa depleción de linfocitos $\mathrm{B}$, ha sido evaluado en el tratamiento del pénfigo al igual que etanercept -antagonista de TNFo-, ambos con resultados promisorios ${ }^{11}$. La dapsona también podría ser útil como ahorrador de corticoides. Por otra parte, la ciclosporina pareciera no tener un rol como ahorrador de corticoides ${ }^{17}$.

Agregar pimecrolimus 1\% tópico al tratamiento con prednisona y azatioprina pareciera ser efectivo $^{20}$. Tacrolimus sistémico ${ }^{21}$, antimaláricos nuevos como la daphentina ${ }^{22}$, administración de altas dosis de péptidos de Dsg ${ }^{11}$, inhibidores de caspasas $^{23}$ y el transplante alogénico de médula ósea ${ }^{24}$ podrían considerarse como alternativas a futuro.

Uno de los problemas del tratamiento del pénfigo son los efectos adversos derivados del mismo.

Es importante considerar que en pacientes que reciban una dosis de prednisona mayor a $16 \mathrm{mg} /$ día por más de $8 \mathrm{sem}$ se debe agregar cotrimoxazol forte trisemanal o cotrimoxazol simple en dosis diaria, para la profilaxis de neumonía por Pneumocystis jiroveci ${ }^{25}$. Otros autores prefieren realizar conteos de linfocitos T CD4 al cabo de un mes de inmunosupresión sólo si ${ }^{1}$ : el conteo total de linfocitos es menor a $600 / \mathrm{mm}^{3}$, 2 si se planea someter al paciente a más de 3 meses de terapia esteroidal $\mathrm{o}^{3}$ si se usan más de $15 \mathrm{mg} /$ día de prednisona o su equivalente; en estos casos y si el conteo de linfocitos T CD4 es menor a 200/ $\mathrm{mm}^{3}$, los autores recomiendan iniciar profilaxis con cotrimoxazol en el esquema antes comentado ${ }^{26}$.

Otra consideración importante cuando se usan tratamientos esteroidales prolongados, es la adición de calcio ( $1.200 \mathrm{mg} /$ día $)$ y vitamina D (800-2.000 U/día) con dosis mayores de $5 \mathrm{mg} /$ día de prednisona por más de 3 meses ${ }^{27,28}$.

\section{Referencias}

1. Bystryn JC, Rudolph JL. Pemphigus. Lancet 2005; 366: 61-73.

2. Murrell DF, Dick S, Ahmed AR, Amagai M, Barnadas MA, Borradori L, et al. Consensus statement on definitions of disease, end points, and therapeutic response for pemphigus. J Am Acad Dermatol 2008; 58: 1043-6.

3. Ribeiro JM, Valenzuela JG, Pham VM, Kleeman L, Barbian KD, Favreau AJ, et al. An insight into the sialotrans- criptome of Simulium nigrimanum, a black fly associated with fogo selvagem in South America. Am J Trop Med Hyg 2010; 82: 1060-75.

4. Mahajan VK, Sharma NL, Sharma RC, Garg G. Twelveyear clinico-therapeutic experience in pemphigus: a retrospective study of 54 cases. Int J Dermatol 2005; 44: 821-7.

5. Ahmed AR, Mohimen A, Yunis EJ, Mirza NM, Kumar $\mathrm{V}$, Beutner $\mathrm{EH}$, et al. Linkage of pemphigus vulgaris antibody to the major histocompatibility complex in healthy relatives of patients. J Exp Med 1993; 177: 41924.

6. Kanwar AJ, De D. Pemphigus in India. Indian J Dermatol Venereol Leprol 2011; 77: 439-49.

7. Bastuji-Garin S, Souissi R, Blum L, Turki H, Nouira R, Jomaa B, et al. Comparative epidemiology of pemphigus in Tunisia and France: unusual incidence of pemphigus foliaceus in young Tunisian women. J Invest Dermatol 1995; 104: 302-5.

8. Yoshida K, Takae Y, Saito H, Oka H, Tanikawa A, Amagai $\mathrm{M}$, et al. Cutaneous type pemphigus vulgaris: a rare clinical phenotype of pemphigus. J Am Acad Dermatol 2005; 52: 839-45.

9. Shinkuma S, Nishie W, Shibaki A, Sawamura D, Ito K, Tsuji-Abe Y, et al. Cutaneous pemphigus vulgaris with skin features similar to the classic mucocutaneous type: a case report and review of the literature. Clin Exp Dermatol 2008; 33: 724-8.

10. Esmaili N, Chams-Davatchi C, Valikhani M, Daneshpazhooh M, Balighi K, Hallaji Z, et al. Pemphigus vulgaris in Iran: a clinical study of 140 cases. Int J Dermatol 2007; 46: 1166-70.

11. $\mathrm{Hu} \mathrm{CH}$, Michel B, Schiltz JR. Epidermal acantholysis induced in vitro by pemphigus autoantibody. An ultrastructural study. Am J Pathol 1978; 90: 345-62.

12. Amagai M. Autoimmunity against desmosomal cadherins in pemphigus. J Dermatol Sci 1999; 20: 92-102.

13. Mahajan R, De D. What is new in autoimmune vesicobullous disorders? Indian J Dermatol Venereol Leprol 2011; 77: 407-12.

14. Aithal V, Kini U, Jayaseelan E. Role of direct immunofluorescence on Tzanck smears in pemphigus vulgaris. Diagn Cytopathol 2007; 35: 403-7.

15. Wiwanitkit V. A systematic metanalysis on diagnostic value of Dsg3 ELISA for pemphigus vulgaris. Indian J Dermatol 2009; 54: 192.

16. Anand V, Khandpur S, Sharma VK, Sharma A. Utility of desmoglein ELISA in the clinical correlation and disease monitoring of pemphigus vulgaris. J Eur Acad Dermatol Venereol 2011 doi: 10.1111/j.14683083.2011.04294.x. 
17. Nair PS, Moorthy PK, Yogiragan K. A study of mortality in dermatology. Indian J Dermatol Venereol Leprol 2005; 71:23-5.

18. Martin LK, Werth VP, Villaneuva EV, Murrell DF. A systematic review of randomized controlled trials for pemphigus vulgaris and pemphigus foliaceus. J Am Acad Dermatol 2011; 64: 903-8.

19. Singh S. Evidence-based treatments for pemphigus vulgaris, pemphigus foliaceus, and bullous pemphigoid: A systematic review. Indian J Dermatol Venereol Leprol 2011; 77: 456-69.

20. Iraji F, Asilian A, Siadat AH. Pimecrolimus 1\% cream in the treatment of cutaneous lesions of pemphigus vulgaris: a double-blind, placebo-controlled clinical trial. J Drugs Dermatol 2010; 9: 684-6.

21. Büsing V, Kern JS, Bruckner-Tuderman L, Hofmann SC. Recalcitrant pemphigus vulgaris responding to systemic tacrolimus. Dermatology 2010; 221: 122-6.

22. Feily A, Reza Fallahi H. Potential utility of daphnetin as a novel treatment for pemphigus vulgaris. G Ital Dermatol Venereol 2010; 145: 557-8.

23. Pacheco-Tovar D, López-Luna A, Herrera-Esparza R,
Avalos-Díaz E.The caspase pathway as a possible therapeutic target in experimental pemphigus. Autoimmune Dis 2011; 2011: 563091.

24. Suslova IM, Theodoropoulos DS, Cullen NA, Tetarnikova MK, Tetarnikov AS, Kolchak NA. Pemphigus vulgaris treated with allogeneic hematopoietic stem cell transplantation following non-myeloablative conditioning. Eur Rev Med Pharmacol Sci 2010; 14: 785-8.

25. Lehman JS, Kalaaji AN. Role of primary prophylaxis for pneumocystis pneumonia in patients treated with systemic corticosteroids or other immunosuppressive agents for immune-mediated dermatologic conditions. J Am Acad Dermatol 2010; 63: 815-23.

26. Sowden E, Carmichael AJ. Autoimmune inflammatory disorders, systemic corticosteroids and pneumocystis pneumonia: a strategy for prevention. BMC Infect Dis 2004; 4: 42.

27. Pereira RM, Carvalho JF, Canalis E. Glucocorticoidinduced osteoporosis in rheumatic diseases. Clinics (Sao Paulo) 2010; 65: 1197-205.

28. Weinstein RS. Clinical practice. Glucocorticoid-induced bone disease. N Engl J Med 2011; 365: 62-70. 